\title{
ORIGINAL ARTICLE \\ Lateral transfers of insertion sequences between Wolbachia, Cardinium and Rickettsia bacterial endosymbionts
}

\author{
O Duron
}

Various bacteria live exclusively within arthropod cells and collectively act as an important driver of arthropod evolutionary ecology. Whereas rampant intra-generic DNA transfers were recently shown to have a pivotal role in the evolution of the most common of these endosymbionts, Wolbachia, the present study show that inter-generic DNA transfers also commonly take place, constituting a potent source of rapid genomic change. Bioinformatic, molecular and phylogenetic data provide evidence that a selfish genetic element, the insertion sequence ISRpe1, is widespread in the Wolbachia, Cardinium and Rickettsia endosymbionts and experiences recent (and likely ongoing) transfers over long evolutionary distances. Although many ISRpe1 copies were clearly expanding and leading to rapid endosymbiont diversification, degraded copies are also frequently found, constituting an unusual genomic fossil record suggestive of ancient ISRpel expansions. Overall, the present data highlight how ecological connections within the arthropod intracellular environment facilitate lateral DNA transfers between distantly related bacterial lineages.

Heredity (2013) 111, 330-337; doi:10.1038/hdy.2013.56; published online 12 June 2013

Keywords: Endosymbiosis; Wolbachia; Cardinium; Rickettsia; arthropod; insertion sequences

\section{INTRODUCTION}

Intracellular bacteria are extremely common and diverse in arthropods (Moran et al., 2008; Wernegreen, 2012). These endosymbionts have a highly specialized lifestyle, replicating exclusively in arthropod cells and depending on maternal transmission to ensure their transmission. While some act as partners that contribute to host adaptation against environmental stresses, some others are subtle parasites that manipulate host reproduction (Werren et al., 2008; Engelstadter and Hurst, 2009). Among their most far-reaching effects is the facilitation of ecological and reproductive isolation of host populations, making endosymbionts important drivers of arthropod evolutionary ecology (Engelstadter and Hurst, 2009; Cordaux et al., 2011).

Endosymbionts originate from a variety of bacterial lineages, typically distantly related, as exemplified by Wolbachia ( $\alpha$-proteobacteria), Rickettsia ( $\alpha$-proteobacteria) and Cardinium (Bacteroidetes), which are each estimated to be present in ca. $5-40 \%$ of arthropod species (Goodacre et al., 2006; Weinert et al., 2007; Duron et al., 2008a; Zug and Hammerstein, 2012). The constitution of their genomes is dynamic, with flexible gene content and rampant intra-generic recombination, indicating accelerated rates of diversification and adaptability (Wu et al., 2004; Klasson et al., 2008; Felsheim et al., 2009; Klasson et al., 2009; Gillespie et al., 2012; Penz et al., 2012; Duplouy et al., 2013). Remarkably, mobile genetic elements represent substantial fractions of these genomes (Bordenstein and Reznikoff, 2005; Wernegreen, 2005; Moran et al., 2008) as exemplified with insertion sequences (ISs), a group of genetically compact transposable elements encoding no functions other than an enzyme that catalyzes their own transposition (Felsheim et al., 2009; Cerveau et al., 2011b). These latter elements are fundamentally selfish DNA parasites, with the capacity to autonomously replicate, proliferating and jumping to other loci within, but also between, genomes (Chandler and Mahillon, 2002; Siguier et al., 2006a). As their mobility and accumulation can alter gene expression, trigger genomic rearrangements and modulate genome size, IS have come to be recognized as major catalysts of changes in endosymbiont genomes (Felsheim et al., 2009; Klasson et al., 2009; Cerveau et al., 2011a).

IS acquisitions following lateral transfer of an existing IS from one bacterial strain to another are common in free-living bacteria but were thought to be rare in endosymbionts as they reside in confined and isolated intracellular environments (Chandler and Mahillon, 2002; Wagner and de la Chaux, 2008). However, the opportunity for coinfections, in which different endosymbionts reside within the same host cell (Weinert et al., 2007; Duron et al., 2008a,b), and their propensity to switch between arthropod hosts (Vavre et al., 1999; Baldo et al., 2008; Jousselin et al., 2012) could have created freely recombining intracellular bacterial communities. This is known as the 'intracellular arena' hypothesis (Bordensteinnd and Wernegreen, 2004; Kent and Bordenstein, 2010). That intra-generic IS transfers occur, and are thus a key factor in shaping endosymbiont evolution, is beyond doubt: phylogenetic evidence indicates that many IS have an extensive history of movement between Wolbachia strains (Cordaux et al., 2008; Cerveau et al., 2011b). Inter-generic IS transfers have, however, rarely been reported (Felsheim et al., 2009) and thus thought to be rare within endosymbiont communities.

Here, I have approached this issue by undertaking an extensive screening for the presence and diversity of ISRpe1, an IS of the IS481 family, in endosymbiont communities inhabiting arthropod cells. When first described, ISRpe1 was found in the tick symbiont 
Rickettsia peacockii in which it was shown to exhibit an intense transpositional activity with several identical copies disseminated along the bacterial chromosome (Simser et al., 2005). Remarkably, the ISRpe1 distribution is not exclusively restricted to Rickettsia as it was recently found in Cardinium, suggesting that this IS could achieve inter-generic lateral transfer (Felsheim et al., 2009). Here, bioinformatic, molecular and phylogenetic investigations were used to further investigate ISRpel activity by tracking its evolutionary dynamics and history.

\section{MATERIALS AND METHODS}

\section{Detection assays}

ISRpe1 sequences were first identified through Basic Local Alignment Search Tool (BLAST) searches against the GenBank database (http://www.ncbi.nlm.nih.gov/genbank/), including the 'microbial genomes' section (which lists microbial genome records in GenBank), and against the IS reference database ISFinder (which provides a list of ISs isolated from eubacteria and archae; Siguier et al., 2006b). The consensus sequence of 44 ISRpel copies from the $R$. peacockii genome (Felsheim et al., 2009) was used as a first query, and the Wolbachia- and Cardinium-ISRpel copies evidenced from the first BLAST search were further used as additional queries in subsequent BLAST searches. BlastN and TBlastX searches were performed with default parameters without low-complexity region filter, using a minimal subject size of $40 \mathrm{bp}$, minimal similarity of $75 \%$, maximal $e$ value of 0.05 , reward of 2 and penalty of 3 .

Second, specific PCR assays were designed to examine the presence of ISRpel in an additional set of bacterial samples including 20 Wolbachia and 9 Cardinium strains identified from 22 insect, 6 arachnid and 1 filarial nematode DNA samples (Supplementary Table S1). For each DNA sample, independent assays for infection by the five most common known endosymbionts, that is, Wolbachia, Cardinium, Rickettsia, Arsenophonus and Spiroplasma, were performed using specific PCR amplifications of $16 S r R N A$ or wsp gene fragments (Supplementary Table S3). Bacterial infection status was further confirmed by direct sequencing. Each DNA sample retained for further analyses was infected by a single bacterial strain. One DNA sample per bacterial strain was finally examined for the presence of ISRpel, except for the Cardinium cPlu strain infecting the spider Holocnemus pluchei, for which more infected individuals were available from a previous study (Stefanini and Duron, 2012). Samples were next tested for ISRpel presence using six independent PCR assays, each using different pairs of primers internal to the ISRpe1-transposase (Tnp) sequence (Supplementary Table S3).

Arthropod DNA (including endosymbiont DNA) was extracted using the DNeasy Blood and Tissue Kit (Qiagen, Hilden, Germany). DNA quality was routinely tested by PCR amplification of a conserved region of mitochondrial COI gene. All the PCR amplifications were performed under the following conditions: initial denaturation at $93{ }^{\circ} \mathrm{C}$ for $3 \mathrm{~min}, 30$ cycles of denaturation $\left(93^{\circ} \mathrm{C}, 30 \mathrm{~s}\right)$, annealing $\left(50-54^{\circ} \mathrm{C}, 30 \mathrm{~s}\right)$, extension $\left(72^{\circ} \mathrm{C}, 1-2\right.$ min depending on primers) and a final extension $\left(72{ }^{\circ} \mathrm{C}, 5 \mathrm{~min}\right)$. The PCR products were electrophoresed in a $1.5 \%$ agarose gel. PCR Direct sequencing of PCR products was done on an ABI Prism 3130 sequencer using the BigDye Terminator Kit (Applied Biosystems, Foster City, CA, USA) after purification with the QIAquick gel extraction kit (Qiagen). The chromatograms were manually inspected and cleaned with Chromas lite (http://www.technelysium.com.au/ chromas_lite.html). Gene features and primers are listed in Supplementary Table S3.

Phylogenetic relationships were evaluated using (i) $16 S$ rRNA gene sequences of Wolbachia, Rickettsia, Cardinium and Amoebophilus strains and (ii) their respective ISRpel sequences whenever present. Phylogenetic relationships of $16 S$ rRNA sequences were evaluated independently for Wolbachia (856 bp), Rickettsia (1526bp) and Cardinium + Amoebophilus (930bp). Phylogenetic analysis of ISRpel sequences was made using $466 \mathrm{bp}$ fragments of Tnp sequences from Wolbachia, Rickettsia, Cardinium and Amoebophilus, as well as using more divergent sequences from other bacterial species: Chitinophaga pinensis (accession number NC013132), Treponema primitia (NC015578), Bradyrhizobium japonicum (NC004463), Burkholderia glumae (NC012721),
Nitrosomonas europaea (NC004757), Actinobacillus pleuropneumoniae (ADOL01000024), Legionella pneumophila (NC006369), Vibrio parahaemolyticus (ACFO01000016), V. vulnificus (NC005140), Pseudoalteromonas haloplanktis (NZADOP01000095), Roseobacter denitrificans (NC008386), Shewanella putrefaciens (CP000681), S. oneidensis (NC004349), Erwinia sp. (CP002124) and E. pyrifoliae (NC012214). The evolutionary model most closely fitting the sequence data was determined using hierarchical likelihood ratio tests and Akaike information criterion with the program MODELTEST [46]. The best-fit approximations were the GTR $+\mathrm{G}$ for Wolbachia and Rickettsia $16 S$ rRNA, TN93 + G for Cardinium + Amoebophilus $16 S$ rRNA and GTR + G + I for ISRpel-Tnp. Phylogenetic analyses were based on maximum likelihood (ML) analyses. A ML heuristic search, using a starting tree obtained by neighbor joining, was conducted in PhyML (Guindon and Gascuel, 2003). Clade robustness was assessed by bootstrap analysis using 1000 replicates.

\section{Isolation of total RNA and RT-PCR}

The expression of ISRpe1-Tnp was examined for the Cardinium cPlu strain infecting $H$. pluchei. Spider total RNA was extracted using NucleoSpin RNA II kits (Macherey-Nagel, Düren, Germany) according to the manufacturer's protocol with the following modifications. RNA was digested for $30 \mathrm{~min}$ using 30 additional DNase I units to avoid genomic DNA contamination. Firststrand complementary DNA (cDNA) synthesis was performed on $100 \mathrm{ng}$ of total RNA using SuperScript II reverse transcriptase (RT) (Invitrogen, Carlsbad, CA, USA) and 85 pmol of 10-nucleotide random primers. cDNA was purified on QIAquick minicolumns (Qiagen) and eluted in $50 \mu \mathrm{l}$ of ddH20. One microliter of cDNA was mixed with ISRpe1 internal primers ISRpe1-f1 and ISRpe1-r1 (Supplementary Table S3), and PCR was run as described above. The absence of genomic DNA contamination was checked by negative $16 S$ rRNA and ISRpel PCR amplifications when RT was omitted. cDNA from Cardinium-uninfected spiders was used as control of expression.

\section{RESULTS}

\section{Distribution of ISRpe1}

Examination of both Genbank and ISfinder databases revealed a wide distribution of ISRpe1. Copies were detected within 26 of 64 completely or partly sequenced genomes of Wolbachia (found in all of 12 genomes), Rickettsia (13/51) and Cardinium (1/1) (Supplementary Table S1). Following original genome annotations, a few ISRpe1 sequences were already referenced, but with different names, as illustrated with ISWpi2 in Wolbachia pipientis ( $w$ Ri strain). Note that the current IS nomenclature system uses the first letter of the genus and the first two letters of the species name followed by a number (Chandler and Mahillon, 2002). Thus, the fact that ISRpe1 copies have received different names does not indicate that they are genetically distinct but that they were primarily described from different bacterial species.

Apart from copies found in full genomes, five Wolbachia-, one Rickettsia- and one Cardinium-ISRpel partial sequences were also identified in short DNA sequences $(<1 \mathrm{~kb})$ found in GenBank (Supplementary Table S1). The presence of ISRpe1 was further assayed through direct PCR detection assays in an additional set of endosymbiont strains, thus indicating ISRpel in 11 out of the 20 tested Wolbachia strains and 5 out of 9 Cardinium strains (Supplementary Table S1).

Overall, ISRpe1 was thus found to be common in Wolbachia, Rickettsia and Cardinium strains from insects and arachnids, including some Rickettsia strains (for example, R. felis and R. massiliae) that also cause vertebrate diseases as part of their life cycle (Supplementary Table S1). It should be noted, however, that the Wolbachia wAna genome available on GenBank (Supplementary Table S1), and which contains ISRpel copies, is suspected to come from a transfer of bacterial DNA into the insect host genome (Dunning Hotopp et al., 2007) and thus might be not considered as a pure Wolbachia 
A

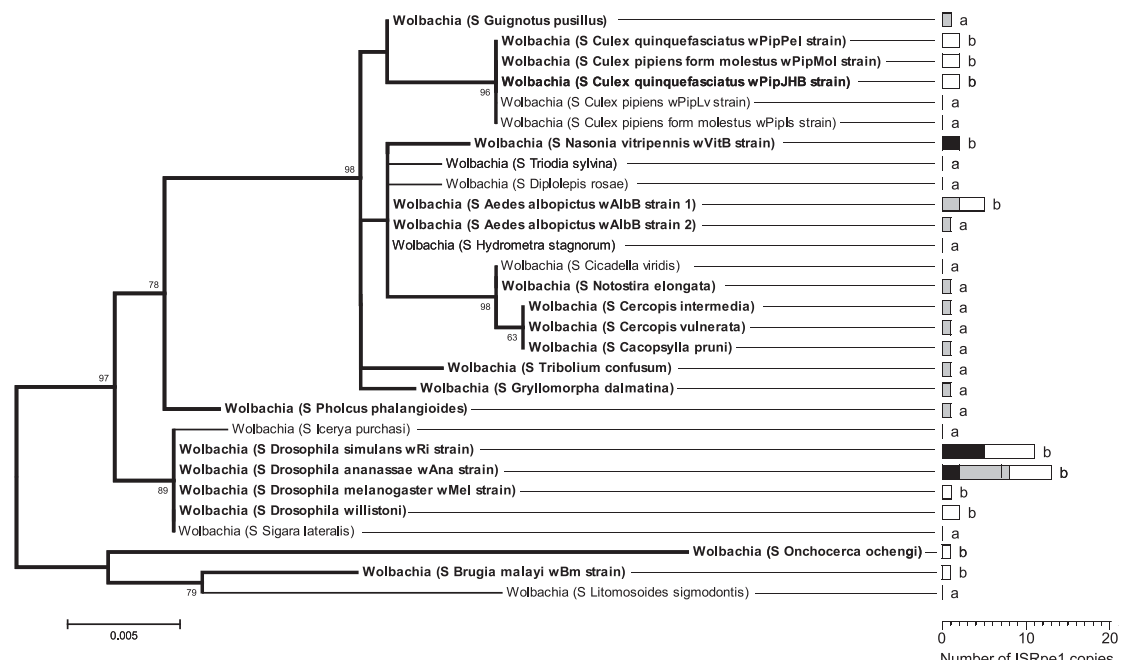

B

B Rickettsia sibinica mongolitimonae (HA-91 strain)

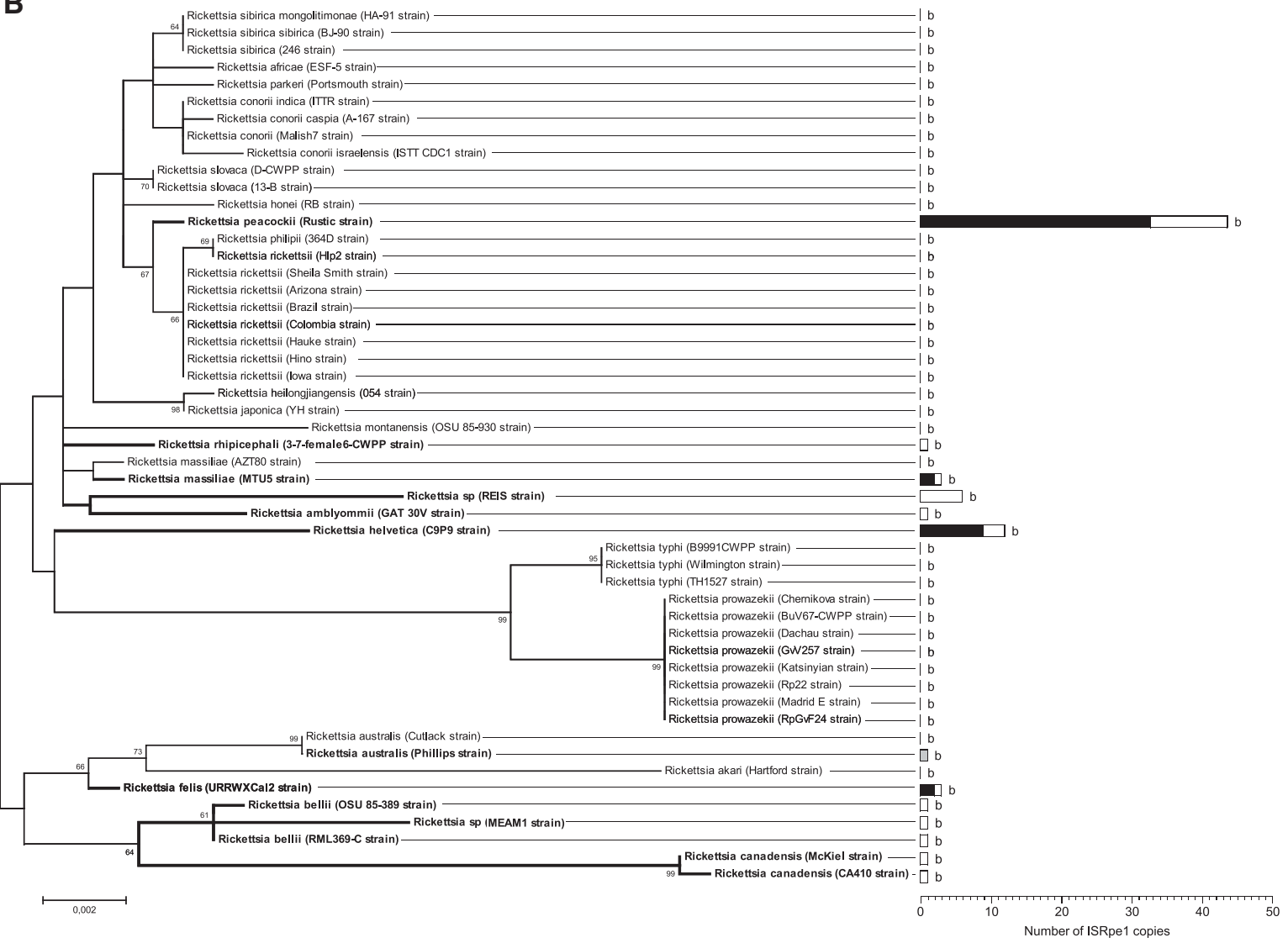

C

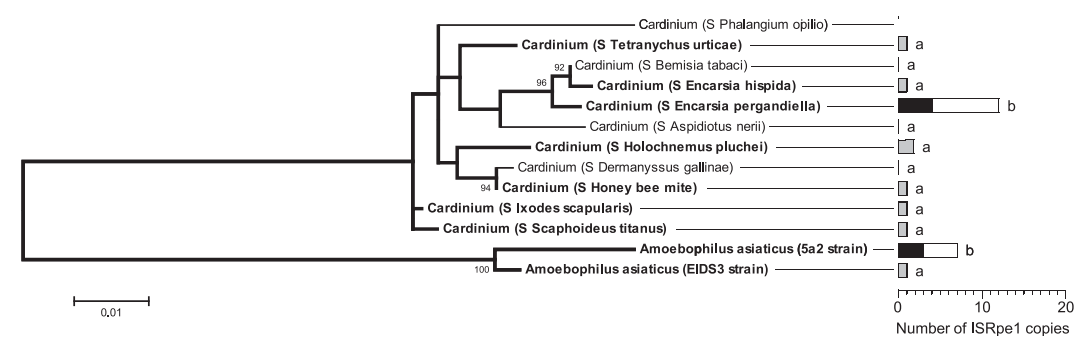


sequence. In addition, other partial ISRpel sequences were found in two strains of Wolbachia present in filarial nematodes and, referenced as ISCaa12, in two strains of Candidatus Amoebophilus asiaticus (Bacteroidetes), an amoebal endosymbiont closely related to Cardinium (Supplementary Table S1).

Overall, ISRpe1 sequences were detected in $50 \%$ of the strains examined (51/102 strains, including the two strains of $A$. asiaticus) with a patchy distribution along the bacterial $16 S r R N A$ phylogeny (Figure 1). ISRpel was, however, not uniformly distributed among bacterial genera (Fisher's exact test, $P<10^{-5}$ ): common in Wolbachia, being detected in 28 of the 37 strains examined (76\%), as well as in Cardinium (7/11;64\%), but rarer in Rickettsia (14/52; 27\%). There are at least two cases ( $R$. massiliae and $R$. australis) for which ISRpe1 has been acquired or lost so recently that it is polymorphic for the presence or absence among closely related strains sharing identical $16 \mathrm{~S}$ rRNA sequences (Supplementary Table S1).

When present, the ISRpel copy number per genome is variable between bacterial strains. Overall, 164 distinct ISRpel copies were found among the 51 positive Wolbachia, Rickettsia, Cardinium and A. asiaticus genomes. The examination of completely sequenced genomes revealed that only one copy per genome was found in some cases (for example, $R$. australis and $R$. rhipicephali) while in other cases more copies were detected, as shown in $R$. massiliae MTU5 (3 copies), Wolbachia $w$ Ri (11 copies) and Cardinium cEper1 (12 copies) (Figure 1 and Supplementary Table S2). An extreme case was found in the $R$. peacockii genome, with 44 copies that account for 3.8\% of total genomic content, as first reported by (Felsheim et al., 2009). No preferential insertion site was found: copies are inserted either in bacterial chromosomes (for example, Wolbachia $w \mathrm{Ri}, \quad R$. massiliae and $R$. peacockii) or in prophages (Wolbachia $w \mathrm{VitB}$ ) or in plasmids (R. felis and R. peacockii).

That ISRpe1 is well transmitted along with bacterial chromosomes was checked through the examination of co-transmission pattern with Cardinium cPlu infection in the marbled cellar spider $H$. pluchei. Ten Cardinium-infected mothers and five uninfected mothers, all carrying eggs, were collected in the field, and, after hatching, 10 spiderlings per clutch were randomly sampled for PCR screening using ISRpe1- and Cardinium-specific primers. All spiderlings $(n=100)$ from infected mothers themselves harbored Cardinium and ISRpe1, whereas no Cardinium infection and no ISRpel copy was found in spiderlings $(n=50)$ from uninfected mothers, showing a clear association of ISRpe1 with Cardinium infection.

\section{Functionality of ISRpe1 copies}

The examination of the ISRpel copies revealed variable lengths ranging from 42-2471 bp (Supplementary Table S2). Of the 164 ISRpe1 copies, the size can, however, not be formally determined (i) in 9 cases for which ISRpel sequences are incomplete as they are located at the end of unassembled contigs (Supplementary Table S2) and (ii) in 26 cases for which only partial ISRpe1 fragments were obtained by PCR amplifications (Supplementary Table S1). The 129 remaining ISRpe1 copies are flanked at both extremities by non-ISRpel sequences, allowing their size to be measured. This revealed a clear bimodal distribution with a straight peak at large size (1100-1300 bp) and a flat peak at small size $(<300 \mathrm{bp}$ ) (Figure 2$)$. As the variable sizes of ISRpe1 copies should result in different transpositional activity, the analysis was further refined by classifying the 129 copies into two groups: potentially functional copies and non-functional copies.

Potentially functional ISRpe1 copies were here defined as full-length copies with intact Tnp genes and flanked by inverted repeat sequences (that are involved in the cleavages and strand transfer reactions leading to IS transposition; Chandler and Mahillon, 2002). Over the 129 ISRpe1 copies examined, 62 (48\%) fit with this definition, being found in Wolbachia (3 genomes), Rickettsia (4), Cardinium (1) and A. asiaticus (1) (Figure 1 and Supplementary Table S2). These potentially functional copies all shared hallmarks of genuine 481 IS family (Chandler and Mahillon, 2002), with a length ranging from $1180-1235$ bp that corresponds to the straight peak in the distribution of copy size (Figure 2). Alignments of their Tnp amino-acid sequences showed $62-100 \%$ identity and revealed the presence of many conserved sites (Supplementary Figure S1). Three of these conserved residues constitute the $\mathrm{N} 2, \mathrm{~N} 3$ and $\mathrm{C} 1$ domains carrying a DDE catalytic triad of amino acids that is crucial for Tnp catalytic activity (Chandler and Mahillon, 2002). All potentially functional ISRpe1-Tnp were also found flanked by 26 nucleotides of inverted repeat sequences to both extremities (Supplementary Table S2). To formally assess ISRpe1 functionality, an RT-PCR analysis was conducted on the Cardinium cPlu strain. The expected 513bp amplicon was detected in total cDNA obtained from all Cardinium-infected spiders while no amplicon was observed using cDNA from Cardinium-uninfected spiders. This establishes that the ISRpe1-Tnp is transcriptionally active in this Cardinium strain, as also shown in R. peacockii (Simser et al., 2005) and A. asiaticus (Schmitz-Esser et al., 2011).

Other ISRpe1 copies (67 of 129) have degraded sequences, and are thus non-functional: 11 contain internal stop codon, disrupting the

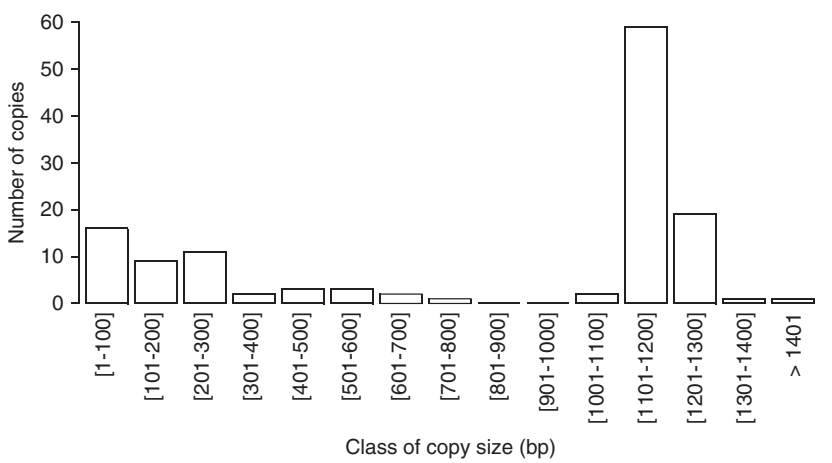

Figure 2 Distribution of ISRpe1 copy size $(n=129)$.

Figure 1 Distribution of ISRpe1 along the 16S rRNA phylogeny of bacterial endosymbionts. (A) Wolbachia phylogeny; (B) Rickettsia phylogeny; and (C) Bacteroidetes phylogeny. The left part of the figure shows the phylogeny of the Wolbachia ( $\alpha$-proteobacteria), Rickettsia ( $\alpha$-proteobacteria), Cardinium (Bacteroidetes) and Amoebophilus (Bacteroidetes) strains via maximum likelihood. Bacterial strains are here identified either by their names (if any) or by the prefix $S$ followed by the proper name of their host. Numbers on branches indicate percentage bootstrap support for major branches (1000 replicates; only bootstrap values of $60 \%$ or more are shown). Bacterial strains harboring ISRpel are shown in bold. The scale bar is in units of substitutions per site. The right part of the figure gives the distribution of ISRpe1 observed with each bacterial strain (black: potentially functional ISRpe1 copies, $n=62$; white: non-functional copies, $n=67$; gray: unknown functional status, $n=26$ ). Methods of detection are indicated by letters (a: specific PCR assays; b: full genome sequencing). 
Tnp-coding sequences, 53 are truncated at either $5^{\prime}$ or $3^{\prime}$ (or both) ends, one shows a $785 \mathrm{bp}$ deletion within the Tnp sequence, and two harbor a large insertion of an exogenous DNA fragment ( $>100 \mathrm{bp}$ )

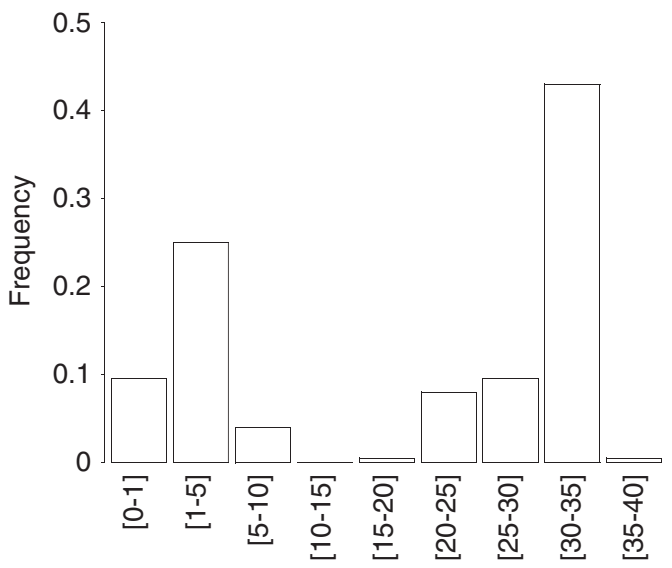

Class of nucleotide divergence (\%)

Figure 3 Frequency distribution of pairwise ISRpe1-Tnp nucleotide divergence. The distribution is based on a total of 1942 pairwise comparisons between Tnp from the 62 potentially functional ISRpe1 copies.
(Figure 1 and Supplementary Table S2). Remarkably, an ISRpe1 copy present in the Wolbachia $w$ Ri genome is disrupted by the insertion of another IS, ISWpil, which is known to be widespread in Wolbachia (Duron et al., 2005; Cordaux et al., 2008; Cordaux, 2008). Nonfunctional ISRpe1 copies are found in 25 genomes, including 11 Wolbachia, 12 Rickettsia, 1 Cardinium and 1 A. asiaticus. In 8 cases, non-functional ISRpe1 and functional copies coexist in the same genomes, as in Cardinium cEper1 (4 functional and 8 non-functional copies) and $R$. peacockii (11 and 33). In the 16 other genomes, however, only non-functional copies are found with, in the most extreme cases, a single degraded short copy as shown in $R$. canadensis (321 bp) or in Wolbachia wUni (42 bp) (Figure 1 and Supplementary Table S2).

\section{Evolutionary history of ISRpe1}

The nucleotide divergence between Tnp pairs from the 62 potentially functional ISRpe1 copies was next used to investigate the tempo of ISRpe1 expansions. If ISRpe1 is of recent origin, this should be reflected in a low nucleotide divergence between copies found in endosymbiont genomes. However, the nucleotide divergence between Tnp pairs showed a bimodal distribution, with a first peak corresponding to identical or nearly identical copies ( $<5 \%$ divergence), indicating a recent expansion of some ISRpel copies, and also a

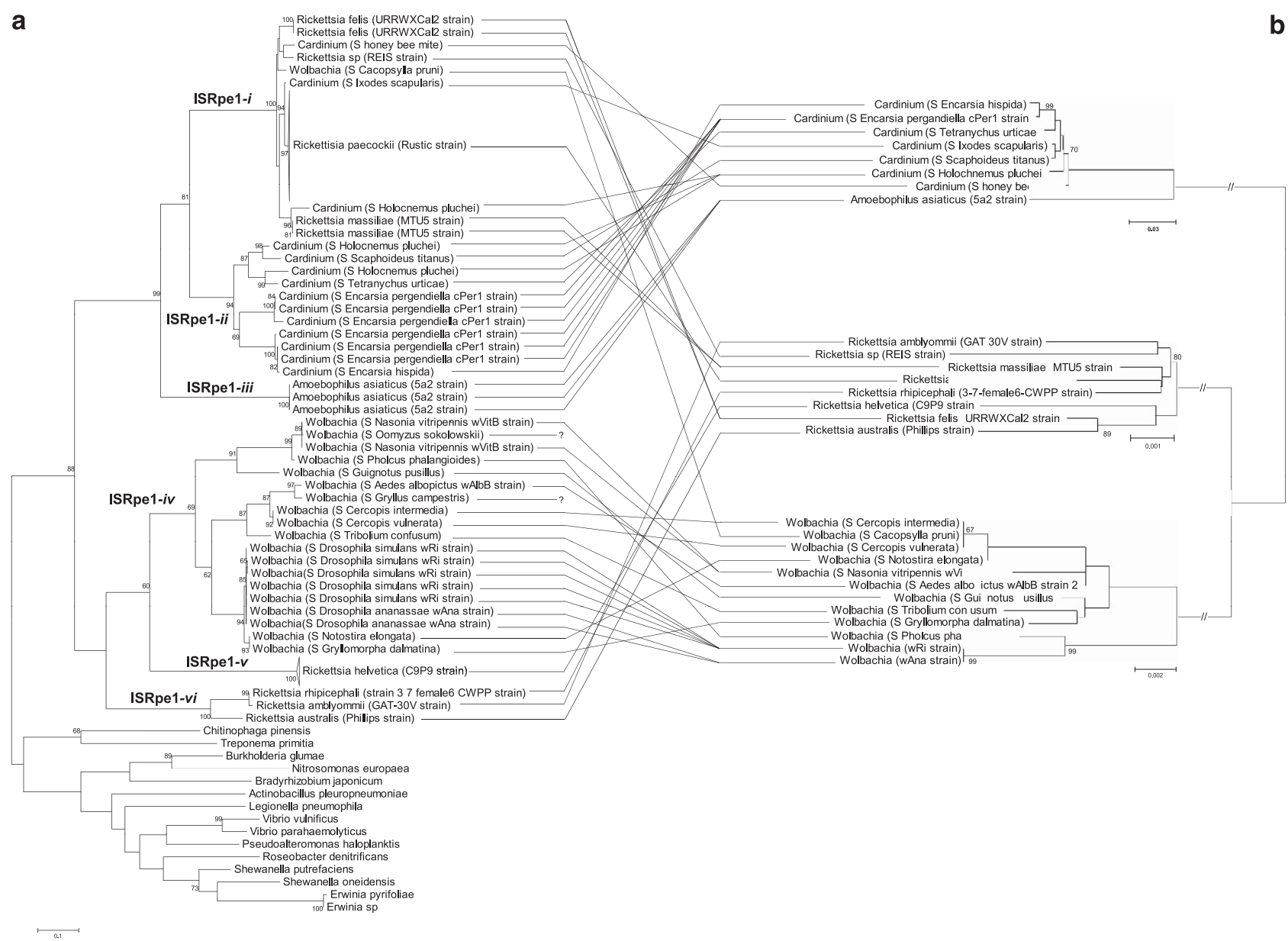

Figure 4 Incongruence between (a) ISRpe1 and (b) 16S rRNA phylogeny trees. Lines connect bacterial strains to their respective ISRpe1 copies. Bacterial strains are here identified either by their names (if any) or by the prefix $S$ followed by the proper name of their host. Names on branches indicate the ISRpe1 clades (ISRpe1-i to -vi). The 16S rRNA sequences were obtained for each bacterial strain, excepted for the Wolbachia infecting Gryllus campestris and Oomyzus sokolowskii (see Supplementary Table S1). Numbers on branches indicate percentage bootstrap support for major branches (1000 replicates; only bootstrap values of $60 \%$ or more are shown). The scale bar is in units of substitutions per site. 


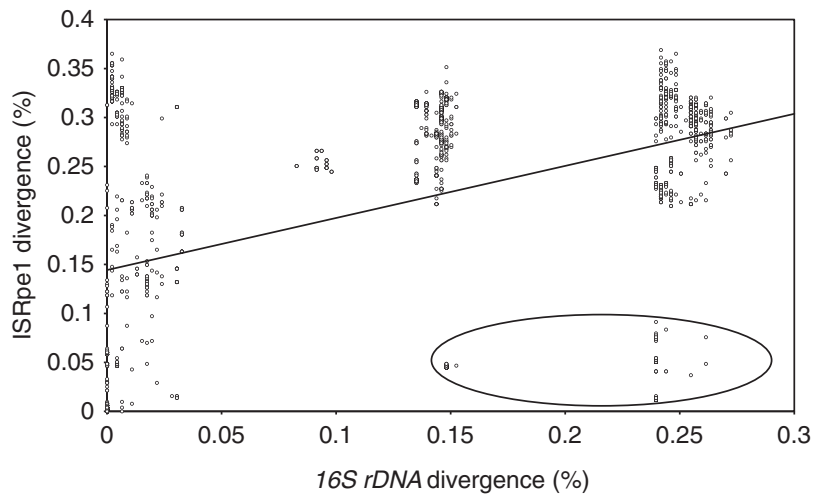

Figure 5 Association between 16S rRNA and ISRpe1 pairwise nucleotide divergence among Wolbachia, Rickettsia, Cardinium and Amoebophilus genomes. The most highly diverged group of bacteria containing very similar ISRpe1 copies is circled, corresponding to the ISRpe1-i clade.

second peak at $20-30 \%$ divergence, indicating the presence of numerous potentially functional ISRpe1 copies that have long since diverged (Figure 3). This pattern does not hold when bacterial genomes are analyzed individually: almost all copies coexisting within a given genome have identical or nearly identical sequences with $<5 \%$ nucleotide divergence, as shown in the Wolbachia $w \mathrm{Ri}$ and $R$. helvetica. A remarkable example is found in the $R$. peacockii genome: the 33 potentially functional copies exhibited a mean of $0.2 \%$ nucleotide divergence, suggesting that only one copy may have contributed to the ISRpel expansion in R. peacockii. A more complex picture was found in the Cardinium $c$ Eper1 and $c$ Plu strains in which more divergent copies have been found. For instance, Tnp from the four potentially functional copies of $c$ Eper1 exhibit a $0.5-11.5 \%$ nucleotide divergence. This pattern suggests either that the primary insertion of ISRpe1 in $c$ Eper1 is so ancient that a substantial degree of diversity has evolved or that divergent copies have been independently acquired by $c$ Eper1 through lateral transfers.

As some ISRpe1 copies have degraded sequences, resulting in short DNA sequences, it was not possible to conduct a phylogenetic analysis based on the Tnp full-length sequences using all ISRpel copies. To analyze a great number of ISRpel sequences, the ISRpel phylogeny was thus constructed using a single 466-bp Tnp fragment from a total of 98 copies from Wolbachia (20 copies), Rickettsia (61), Cardinium (14) and A. asiaticus (3), and from 15 other bacteria in which closest ISRpe1 relatives were found. All the ISRpel copies proved to be phylogenetically related and clearly delineate a monophyletic group confined to bacterial endosymbionts (Figure 4a). The ISRpel group can, however, be divided into six well-supported clades (ISRpe1-i to $-v i$ hereafter). The first of these, ISRpe1-i, is a generalist clade with copies found in Rickettsia, Cardinium and Wolbachia (Figures 4a and b). The typical signature of a recent lateral transfer is here found with distantly related bacterial strains harboring ISRpel copies with low nucleotide divergence (Figure 5). For instance, the Wolbachia strain infecting the psyllid Cacopsylla pruni harbor an ISRpel copy more similar to copies found in Rickettsia and Cardinium than those found in other Wolbachia strains. Additional PCR screening did not reveal other endosymbionts (that is, Cardinium, Rickettsia, Arsenophonus and Spiroplasma) than Wolbachia in C. pruni, showing that this ISRpel copy is associated with Wolbachia rather than with Rickettsia and Cardinium. Another remarkable example is the presence of nearly identical ISRpe1 copies in the Rickettsia REIS strain and one
Cardinium strain, both infecting the deer tick Ixodes scapularis: this pattern suggests that ISRpe1 moves among bacteria that share the same host species, that is, that are ecologically interconnected.

The five other clades (ISRpe1-ii to -vi) show a very different pattern, each being restricted to a single bacterial genus: ISRpe1-ii contains only ISRpe1 copies from Cardinium, ISRpe1-iii from $A$. asiaticus, ISRpe1-iv from Wolbachia, and ISRpe1-v and $-v i$ from Rickettsia (Figures 4a and b). Within each of these five clades, genetically identical ISRpel copies can be present in closely related bacterial strains, as shown with the $w \mathrm{Ri}$ and $w$ Ana Wolbachia strains, suggesting codivergence of ISRpe1 with bacterial genomes. There are, however, many disparities between ISRpel and bacterial $16 \mathrm{~S} r R N A$ phylogenies, as exemplified by the Wolbachia $w \mathrm{Ri}$ and the Wolbachia infecting Gryllomorpha dalmatina, that are not closely related but share almost identical ISRpe1 copies (Figures $4 \mathrm{a}$ and b). This rather suggests that repeated lateral transfers of ISRpe1 have also occurred within these clades.

The ISRpel copies found within each bacterial genus are thus clearly polyphyletic: the Wolbachia-ISRpel copies were found in the ISRpe1- $i$ and -iv clades, the Cardinium-ISRpel in the ISRpe1-i and -ii clades, and the Rickettsia-ISRpel in the ISRpe1-i, $-v$ and $-v i$ clades (only Amoebophilus-ISRpe1 copies are found in a single clade, ISRpe1-iii). As a result, highly divergent ISRpe1 copies, with more than $20 \%$ nucleotide divergence, are commonly found in closely related bacteria, suggesting frequent lateral transfers (Figure 5).

\section{DISCUSSION}

The global incidence of ISRpe1 was $50 \%$ for arthropod endosymbionts. When originally described, ISRpe1 had only been found in the Rickettsia genus, but a wide range of ISRpel sequences have now been found in other endosymbionts, with more than two-thirds of Wolbachia and Cardinium genomes harboring partial or complete (and potentially functional) copies of this selfish genetic element. Some of these copies have undergone a recent and intense transpositional activity that has led to massive intragenomic expansion, as best shown with the 44 almost identical ISRpe1 copies found in the $R$. peacockii genome. This activity may also contribute to the diversification of endosymbionts, with closely related endosymbiont strains that differ in the pattern of the presence/absence of ISRpe1, as shown with the tick endosymbiont $R$. massiliae.

Apart from its propensity for intragenomic spread, ISRpel also often crosses species boundaries to enter new genomes. The patchy distribution of ISRpe1, as well as the presence of closely related copies in unrelated endosymbionts, and the rarity of codivergence between ISRpe1 and bacterial genomes, indicates that lateral transfers have repeatedly occurred recently, thus being a key factor in dictating ISRpel incidence within endosymbiont communities. As first posited by the 'intracellular arena' hypothesis (Bordenstein and Wernegreen, 2004; Kent and Bordenstein, 2010), the frequent co-occurrence of divergent endosymbionts within the same arthropod cells (Weinert et al., 2007; Duron et al., 2008a,b) has likely facilitated ISRpe1 transfers by creating physical proximity. Remarkably, the case of Cardinum and Rickettsia strains inhabiting tick cells and sharing nearly identical copies is representative of this process.

The presence of ISRpe1 in both Bacteroidetes and $\alpha$-proteobacteria is also of particular interest as it implies that IS transfers across exceptionally large phylogenetic distances occur. Distant DNA lateral transfers are usually thought to be rare in bacteria as a variety of barriers are known (Thomas and Nielsen, 2005; Wagner and de la Chaux, 2008). Notably, IS typically lack the machinery for lateral transfer and they need autonomous extrachromosomal elements, such 
as plasmids and bacteriophages, to serve as shuttles (Leclercq and Cordaux, 2011; Leclercq et al., 2012). In some cases, ISRpe1 transfers might be thus facilitated by the presence of WO bacteriophages that commonly jump between Wolbachia strains (Bordenstein and Wernegreen, 2004; Chafee et al., 2010; Atyame et al., 2011). Occasionally, WO bacteriophages may also undergo distant lateral transfer, as recently reported in Rickettsia (Ishmael et al., 2009), and then introduce ISRpe1 to recipient endosymbionts phylogenetically distant from donor strain. In addition, the presence of plasmids in many Cardinium and Rickettsia strains (Ogata et al., 2005; Penz et al., 2012) might serve as shuttles for transferring ISRpe1 among strains.

Given that ISRpe1 is mostly associated with arthropod endosymbionts, the occurrence of functional ISRpel copies in an amoebal endosymbiont, A. asiaticus, is all the more surprising. This might reflect that the ancestors of these endosymbionts shared the same ecological niche at some point in evolutionary history. Amoebae are believed to have contributed to the development of key features for survival in eukaryotic host cells by Cardinium and Rickettsia progenitors (Bordenstein and Reznikoff, 2005; Molmeret et al., 2005; Weinert et al., 2009; Penz et al., 2012). For instance, many genes of $R$. bellii are more related to amoebal symbionts than to other Rickettsia, an observation compatible with an ancestor of $R$. bellii infecting amebas and exchanging genes with other amoebal symbionts (Ogata et al., 2006). Similarly, the ISRpel distribution pattern also indicates an ancient and joint evolutionary history of arthropod and amoebal endosymbionts, suggesting that the ISRpel acquisition by Wolbachia, Cardinium and Rickettsia could have predated their adaptation to arthropods.

An examination of ISRpe1 nucleotide diversity supports this latter hypothesis because it showed that this element is not of recent origin: first, the presence of highly diverged potentially functional copies and second, the identification of a large number of degraded copies that constitute an important genomic fossil record of past ISRpe1 expansions indicates an exceptionally long evolutionary history. However, the ISRpe1 transpositional activity was probably not constant over time, as suggested by the bimodal distribution of pairwise nucleotide divergence. Most ISRpel copies may have been generated in at least two major periods of intense transpositional activity, one ancient and another recent. This could be a widespread feature as several IS families in Wolbachia were recently shown to have the same pattern of transpositional activity (Cerveau et al., 2011b). Endosymbiont IS, including ISRpe1, may thus experiment cyclic expansion separated by periods of low transpositional activity, possibly leading to extinction in some genomes, as previously suggested for diverse IS (Wagner, 2006; Wagner et al., 2007). After an IS enters a genome, its copy number expands rapidly through transposition genera but, through natural selection acting on the whole genome, the IS may further become extinct. The presence of degraded copies in diverse genomes, without evidence for the presence of functional copies, confirms that ISRpe1 may locally become extinct. In this scenario, periodic lateral transfers are thus crucial for the long-term maintenance of ISRpe1 in endosymbiont genomes.

The dynamic of ISRpel insertion is also likely to affect endosymbiont evolution at both genome and phenotype levels. The comparison between $R$. peacockii and its nearest relative, $R$. rickettsii, is informative: multiple ISRpel copies are points of homology for recombination, resulting in numerous deletions, massive genomic rearrangements and loss of gene synteny (Felsheim et al., 2009). Some ISRpe1 insertions were further demonstrated to disrupt coding sequences and extinct gene expression, including those of genes underlying pathogenesis of Rickettsia against vertebrates (Simser et al., 2005). The presence of ISRpe1 may thus create variability in hostadaptation strategies, and then affect the ability of endosymbiont variants to invade arthropod populations.

Arthropod intracellular environments have thus contributed to create a recombining endosymbiont community with mosaic genomes, as previously stated for the Wolbachia genus (Bordenstein and Reznikoff, 2005; Klasson et al., 2009). The frequent ISRpel lateral transfers pose, however, the question of the underlying transmission mechanisms: because IS lack the machinery for lateral transfer, endoymbionts should exchange more genetic material than ISRpe1. Precisely, this pattern has occasionally been reported from recent genomic studies (Ishmael et al., 2009; Penz et al., 2012; Duplouy et al., 2013). What is yet to be established is the extent of these DNA exchanges, especially across large phylogenetic distances, and whether this leads to important innovations in endosymbionts.

\section{DATA ARCHIVING}

New sequence data have been submitted to GenBank (accession numbers KC677578-KC677613; see Supplementary Table S1 (Supporting information) for details).

\section{CONFLICT OF INTEREST}

The author declares no conflict of interest.

\section{ACKNOWLEDGEMENTS}

I am deeply grateful to H Henri and F Vavre for helpful discussion; A Aebi, D Bordat, B Gatimel, L Gavotte and D Papura for providing samples; A Berthomieu, P Makoundou and S Unal for technical help; and four anonymous referees for their comments. All sequence and morphological data were obtained on the Environmental Genomic Platform of the IFR Montpellier-Environnement-Biodiversite. This is contribution 2013.nx of the Institut des Sciences de l'Evolution de Montpellier (UMR 5554 CNRSUniversité Montpellier 2).

Atyame C, Delsuc F, Pasteur N, Weill M, Duron O (2011). Diversification of Wolbachia endosymbiont in the Culex pipiens mosquito. Mol Biol Evol 28: 2761-2772.

Baldo L, Ayoub NA, Hayashi CY, Russell JA, Stahlhut JK, Werren JH (2008). Insight into the routes of Wolbachia invasion: high levels of horizontal transfer in the spider genus Agelenopsis revealed by Wolbachia strain and mitochondrial DNA diversity. Mol Ecol 17: 557-569.

Bordenstein SR, Reznikoff WS (2005). Mobile DNA in obligate intracellular bacteria. Nat Rev Microbiol 3: 688-699.

Bordenstein SR, Wernegreen JJ (2004). Bacteriophage flux in endosymbionts (Wolbachia): infection frequency, lateral transfer, and recombination rates. Mol Biol Evol 21 $1981-1991$

Cerveau N, Leclercq S, Bouchon D, Cordaux R (2011a). Evolutionary dynamics and genomic impact of prokaryote transposable elements. In: Pontarotti P (ed.) Evolutionary Biology - Concepts, Biodiversity, Macroevolution and Genome Evolution. SpringerVerlag: Berlin, Heidelberg, Germany, pp 291-312.

Cerveau N, Leclercq S, Leroy E, Bouchon D, Cordaux R (2011b). Short- and long-term evolutionary dynamics of bacterial insertion sequences: insights from Wolbachia endosymbionts. Genome Biol Evol 3: 1175-1186.

Chafee ME, Funk DJ, Harrison RG, Bordenstein SR (2010). Lateral phage transfer in obligate intracellular bacteria (Wolbachia): verification from natural populations. Mo Biol Evol 27: 501-505.

Chandler M, Mahillon J (2002). Insertion sequences revisited. In: Craig NL, Gellert M, Lambowitz AM (eds) Mobile DNA II. ASM Press: Washington (DC), USA, pp 305-366. Cordaux R (2008). ISWpi1 from Wolbachia pipientis defines a novel group of insertion sequences within the IS5 family. Gene 409: 20-27.

Cordaux R, Bouchon D, Greve P (2011). The impact of endosymbionts on the evolution of host sex-determination mechanisms. Trends Genet 27: 332-341.

Cordaux R, Pichon S, Ling A, Pérez P, Delaunay C, Vavre F et al. (2008). Intense transpositional activity of insertion sequences in an ancient obligate endosymbiont. $\mathrm{Mol}$ Biol Evol 25: 1889-1896. 
Dunning Hotopp JC, Clark ME, Oliveira DC, Foster JM, Fischer P, Muñoz Torres MC et al. (2007). Widespread lateral gene transfer from intracellular bacteria to multicellular eukaryotes. Science 317: 1753-1756.

Duplouy A, Iturbe-Ormaetxe I, Beatson SA, Szubert JM, Brownlie JC, McMeniman CJ et al. (2013). Draft genome sequence of the male-killing Wolbachia strain wBoll reveals recent horizontal gene transfers from diverse sources. BMC Genomics 14: 20.

Duron O, Bouchon D, Boutin S, Bellamy L, Zhou L, Engelstädter J et al. (2008a). The diversity of reproductive parasites among arthropods: Wolbachia do not walk alone. BMC Biol 6: 27.

Duron O, Hurst GD, Hornett EA, Josling JA, Engelstadter J (2008b). High incidence of the maternally inherited bacterium Cardinium in spiders. Mol Ecol 17: 1427-1437.

Duron O, Lagnel J, Raymond M, Bourtzis K, Fort P, Weill M (2005). Transposable element polymorphism of Wolbachia in the mosquito Culex pipiens: evidence of genetic diversity, superinfection and recombination. Mol Ecol 14: 1561-1573.

Engelstadter J, Hurst GDD (2009). The ecology and evolution of microbes that manipulate host reproduction. Ann Rev Ecol Evol Systematics 40: 127-149.

Felsheim RF, Kurtti TJ, Munderloh UG (2009). Genome sequence of the endosymbiont Rickettsia peacockii and comparison with virulent Rickettsia rickettsii: identification of virulence factors. PLoS One 4: e8361.

Gillespie JJ, Joardar V, Williams KP, Driscoll T, Hostetler JB, Nordberg E et al. (2012). A Rickettsia genome overrun by mobile genetic elements provides insight into the acquisition of genes characteristic of an obligate intracellular lifestyle. J Bacterio/ 194: 376-394.

Goodacre SL, Martin OY, Thomas CF, Hewitt GM (2006). Wolbachia and other endosymbiont infections in spiders. Mol Ecol 15: 517-527.

Guindon S, Gascuel O (2003). A simple, fast, and accurate algorithm to estimate large phylogenies by maximum likelihood. Syst Biol 52: 696-704.

Ishmael N, Hotopp JC, loannidis P, Biber S, Sakamoto J, Siozios S et al. (2009). Extensive genomic diversity of closely related Wolbachia strains. Microbiology 155: 2211-2222.

Jousselin E, Coeur d'Acier A, Vanlerberghe-Masutti F, Duron O (2012). Evolution and diversity of Arsenophonus endosymbionts in aphids. Mol Ecol 22: 260-270.

Kent BN, Bordenstein SR (2010). Phage WO of Wolbachia: lambda of the endosymbiont world. Trends Microbiol 18: 173-181.

Klasson L, Walker T, Sebaihia M, Sanders MJ, Quail MA, Lord A et al. (2008). Genome evolution of Wolbachia strain wPip from the Culex pipiens group. Mol Biol Evol 25: 1877-1887.

Klasson L, Westberg J, Sapountzis P, Näslund K, Lutnaes Y, Darby AC et al. (2009). The mosaic genome structure of the Wolbachia wRi strain infecting Drosophila simulans. Proc Natl Acad Sci USA 106: 5725-5730.

Leclercq S, Cordaux R (2011). Do phages efficiently shuttle transposable elements among prokaryotes? Evolution 65: 3327-3331.

Leclercq S, Gilbert C, Cordaux R (2012). Cargo capacity of phages and plasmids and other factors influencing horizontal transfers of prokaryote transposable elements. Mob Genet Elements 2: 115-118.

Molmeret M, Horn M, Wagner M, Santic M, Abu Kwaik Y (2005). Amoebae as training grounds for intracellular bacterial pathogens. Appl Environ Microbiol 71: 20-28.

Moran NA, McCutcheon JP, Nakabachi A (2008). Genomics and evolution of heritable bacterial symbionts. Ann Rev Genet 42: 165-190.
Ogata H, La Scola B, Audic S, Renesto P, Blanc G, Robert C et al. (2006). Genome sequence of Rickettsia bellii illuminates the role of amoebae in gene exchanges between intracellular pathogens. PLoS Genet 2: e76.

Ogata H, Renesto P, Audic S, Robert C, Blanc G, Fournier PE et al. (2005). The genome sequence of Rickettsia felis identifies the first putative conjugative plasmid in an obligate intracellular parasite. PLoS Bio/ 3: e248.

Penz T, Schmitz-Esser S, Kelly SE, Cass BN, Müller A, Woyke T et al. (2012). Comparative genomics suggests an independent origin of cytoplasmic incompatibility in Cardinium hertigii. PLoS Genet 8: e1003012.

Schmitz-Esser S, Penz T, Spang A, Horn M (2011). A bacterial genome in transition-an exceptional enrichment of IS elements but lack of evidence for recent transposition in the symbiont Amoebophilus asiaticus. BMC Evol Biol 11: 270.

Siguier P, Filee J, Chandler M (2006a). Insertion sequences in prokaryotic genomes. Curr Opin Microbiol 9: 526-531.

Siguier P, Perochon J, Lestrade L, Mahillon J, Chandler M (2006b). ISfinder: the reference centre for bacterial insertion sequences. Nucleic Acids Res 34: D32-D36.

Simser JA, Rahman MS, Dreher-Lesnick SM, Azad AF (2005). A novel and naturally occurring transposon, ISRpe1 in the Rickettsia peacockii genome disrupting the rickA gene involved in actin-based motility. Mol Microbiol 58: 71-79.

Stefanini A, Duron O (2012). Exploring the effect of the Cardinium endosymbiont on spiders. J Evol Biol 25: 1521-1530.

Thomas CM, Nielsen KM (2005). Mechanisms of, and barriers to, horizontal gene transfer between bacteria. Nat Rev Microbio/ 3: 711-721.

Vavre F, Fleury F, Lepetit D, Fouillet P, Bouletreau M (1999). Phylogenetic evidence for horizontal transmission of Wolbachia in host-parasitoid associations. Mol Biol Evol 16: 1711-1723.

Wagner A (2006). Periodic extinctions of transposable elements in bacterial lineages: evidence from intragenomic variation in multiple genomes. Mol Biol Evol 23 : 723-733.

Wagner A, de la Chaux N (2008). Distant horizontal gene transfer is rare for multiple families of prokaryotic insertion sequences. Mol Genet Genomics 280: 397-408.

Wagner A, Lewis C, Bichsel M (2007). A survey of bacterial insertion sequences using IScan. Nucleic Acids Res 35: 5284-5293.

Weinert LA, Tinsley MC, Temperley M, Jiggins FM (2007). Are we underestimating the diversity and incidence of insect bacterial symbionts? A case study in ladybird beetles. Biol Lett 3: 678-681.

Weinert LA, Werren JH, Aebi A, Stone GN, Jiggins FM (2009). Evolution and diversity of Rickettsia bacteria. BMC Biol 7: 6.

Wernegreen JJ (2005). For better or worse: genomic consequences of intracellular mutualism and parasitism. Curr Opin Genet Dev 15: 572-583.

Wernegreen JJ (2012). Endosymbiosis. Curr Biol 22: R555-R561.

Werren JH, Baldo L, Clark ME (2008). Wolbachia: master manipulators of invertebrate biology. Nat Rev Microbiol 6: 741-751.

Wu M, Sun LV, Vamathevan J, Deboy R, Brownlie JC, McGraw EA et al. (2004). Phylogenomics of the reproductive parasite Wolbachia pipientis wMel: a streamlined genome overrun by mobile genetic elements. PLoS Biol 2: E69.

Zug R, Hammerstein P (2012). Still a host of hosts for Wolbachia: analysis of recent data suggests that $40 \%$ of terrestrial arthropod species are infected. PLoS One 7: e38544.

Supplementary Information accompanies this paper on Heredity website (http://www.nature.com/hdy) 